\title{
Modelos matemáticos para problemas de dimensionamento de lotes com restrições de capacidade e custos de transporte
}

\author{
Mathematical models for capacitated lot sizing problems \\ with transportation costs
}

\author{
Flavio Molina ${ }^{1}$ \\ Reinaldo Morabito' \\ Silvio Alexandre de Araujo ${ }^{2}$
}

\begin{abstract}
Resumo: Neste trabalho estudam-se problemas de dimensionamento de lotes em que as decisões clássicas do dimensionamento dos lotes de produção são tomadas considerando também os custos do transporte dos produtos fabricados. Estes custos estão associados ao número de paletes ou caminhões necessários para empacotar e/ou transportar os produtos produzidos desde a fábrica até o depósito. São consideradas três extensões de um modelo de programação inteira mista, apresentado na literatura, representando casos mais gerais que ocorrem na prática. Estes modelos são testados e avaliados usando um pacote de otimização. Também é apresentado um método heurístico de resolução, baseado em relaxação Lagrangiana, para uma das extensões propostas.
\end{abstract}

Palavras-chave: Dimensionamento de lotes. Custos de transporte. Programação inteira mista.

\begin{abstract}
In this paper, capacitated lot sizing problems in which the classical lot sizing decisions are made considering the transportation costs of the manufactured products were studied. These costs are related to the necessary number of pallets or trucks to pack and/or transport the products from the factory to the warehouse. Three extensions of a mixed integer linear programming model from the literature are considered, representing general cases that are commonly found in companies. These models are tested and evaluated using an optimization package, and a Lagrangian heuristic was developed for one of the extensions proposed.
\end{abstract}

Keywords: Lot sizing. Transportation cost. Integer mixed programming.

\section{Introdução}

O problema de dimensionamento de lotes pode ser visto como um problema de otimização da produção, em que o objetivo é programar a quantidade de cada item a ser produzida em uma ou várias máquinas, em cada período de um horizonte de tempo, de modo a atender à demanda de cada item e otimizar alguns objetivos de desempenho. Um planejamento eficiente em geral envolve vários fatores, tais como o tempo disponível para produção, a disponibilidade de insumos, o atendimento à demanda e as preparações de máquinas. Cada um destes fatores tem influência nos custos e no desempenho do plano de produção. Revisões recentes para o problema de dimensionamento de lotes podem ser encontradas, por exemplo, em Karimi, Ghomi e Wilson (2003), Brahimi et al. (2006), Jans e Degraeve (2007, 2008) e Robison, Narayananb e Sahinc (2009).
Vários trabalhos vêm apontando que uma melhoria na qualidade das decisões pode ser obtida considerando diferentes fatores do processo produtivo de maneira integrada. Neste sentido, diversas pesquisas têm sido realizadas considerando a integração do problema de dimensionamento de lotes com outros problemas do setor produtivo. Alguns exames sobre tais integrações são encontrados em, por exemplo, Drexl e Kimms (1997) e Jans e Degraeve (2008). De acordo com Jans e Degraeve (2008), as diferentes aplicações industriais têm feito surgir diferentes extensões dos problemas clássicos de dimensionamento de lotes considerando diferentes tipos de integrações.

Os custos considerados nos problemas de dimensionamento de lotes geralmente se limitam aos custos de produção, estocagem, preparação e horas extras. Com isso, desconsideram-se custos que

\footnotetext{
${ }^{1}$ Departamento de Engenharia de Produção, Universidade Federal de São Carlos - UFSCar, Rod. Washington Luís, Km 235, CEP 13565-905, São Carlos, SP, Brasil, e-mail: flaviomolinabr@yahoo.com.br; morabito@ufscar.br

2 Departamento de Ciências da Computação e Estatística, Universidade Estadual Paulista - UNESP, Rua Cristóvão Colombo, 2265, CEP 15054-000, São José do Rio Preto, SP, Brasil, e-mail: saraujo@ibilce.unesp.br
} 
podem ser relevantes para a produção e logística. Por exemplo, em algumas empresas, os produtos finais são embalados e colocados em paletes, para serem estocados e transportados ao longo da cadeia logística até os clientes. O transporte das fábricas até os depósitos pode gerar um custo que está diretamente relacionado ao dimensionamento de lotes. Custos de transporte podem ter comportamentos diferentes em relação ao lote, por exemplo, em uma economia de escala, a alta produção dos itens pode tornar os custos de transporte por produto mais barato. De acordo com Norden e Velde (2005), embora os custos de transporte possam representar mais de $50 \%$ do custo logístico total de um produto, em geral eles são praticamente negligenciados na modelagem dos problemas de dimensionamento de lotes. Vroblefski, Ramesh e Zionts (2000) afirmam que um dos maiores custos em sistemas de distribuição é o custo de transporte e, segundo os autores, este custo tende a ser dependente do volume de produtos transportados.

Os trabalhos, a seguir, tratam de uma maneira integrada o problema de produção e distribuição. Uma revisão geral de problemas integrados de produção e distribuição pode ser encontrada em Erenguç, Simpson e Vakharia (1999) e Rizk e Martel (2001), e uma revisão que considera extensões do problema clássico de dimensionamento de lotes para problemas com custos de transportes aparece em Bertazzi e Speranza (1999). No problema estudado em Lee, Han e Cho (2005), os autores consideram que a capacidade de transporte está associada ao número de contêineres utilizados pelos itens; logo, o objetivo é minimizar o número de contêineres utilizados, uma vez que os custos logísticos são proporcionais a esse número. Os autores propõem uma heurística baseada na representação de fluxos em redes do problema. Em Vroblefski, Ramesh e Zionts (2000) é apresentado um modelo e um algoritmo para tratar um problema de dimensionamento de lotes e distribuição, com um único produto, múltiplos armazéns e horizonte de tempo infinito. Além disso, são considerados os custos de estoque, os custos de transporte e a demanda contínua.

Em Norden e Velde (2005), os custos de carga dependem do tipo de contrato estabelecido com o operador logístico, ou seja, não são somente dependentes do volume de produtos a ser transportado. $\mathrm{O}$ modelo proposto pelos autores considera um problema prático de uma empresa europeia com flutuações mensais de distribuição de produtos entre sua fábrica e seus armazéns. A empresa negocia um contrato de longo prazo com a transportadora, em que um custo fixo por período é associado ao transporte dos itens; em contrapartida, um limite de paletes ou contêineres é disponibilizado com custo unitário mais baixo que o custo padrão. O número limite de paletes ou contêineres é estipulado com base numa previsão de demanda. Caso a empresa necessite de um número maior destas unidades em um período, novas unidades podem ser utilizadas; no entanto, seus custos unitários serão mais elevados. O objetivo é minimizar os custos de produção e de distribuição dos itens desde a fábrica até os armazéns, e, para tanto, os autores propõem uma heurística Lagrangiana. Neste problema, os custos de transporte pesam a favor de aumentar preparações e reduzir estoques. Este comportamento ocorre porque os custos de transporte forçam a produção de um mix de produto maior para um melhor aproveitamento dos paletes ou contêineres. Além disso, para evitar que sejam utilizados paletes ou contêineres caros num determinado período, os custos de transportes forçam que sejam feitas mais preparações e menos estoques.

Recentemente, em Molina et al. (2009) foi proposto um método heurístico para o problema apresentado em Norden e Velde (2005), baseado em relaxação Lagrangiana e Lagrangiana/surrogate. Testes computacionais mostraram que, para este problema, as relaxações Lagrangiana e Lagrangiana/surrogate são equivalentes quanto à qualidade das soluções encontradas; no entanto, o limitante da relaxação Lagrangiana/surrogate é obtido num menor número de iterações. Este resultado está de acordo com os resultados de outros trabalhos da literatura, como, por exemplo, em Senne e Lorena (2000), que trataram do problema das p-medianas. Molina et al. (2009) também propuseram uma extensão do modelo de Norden e Velde (2005) considerando atraso na entrega da demanda e restrições de capacidade de produção. As heurísticas Lagrangiana e Lagrangiana/surrogate também foram adaptadas para o modelo estendido.

No presente trabalho, outras extensões dos modelos propostos em Norden e Velde (2005) e Molina et al. (2009), representando casos mais gerais que ocorrem na prática, são analisadas, bem como é aplicado um método de solução baseado em relaxação Lagrangiana para uma das extensões propostas. Na próxima seção, os modelos estendidos são apresentados e, na Seção 3, é feita uma análise computacional destes modelos utilizando a linguagem de modelagem AMPL e o software de otimização CPLEX. Na Seção 4, é apresentada a heurística Lagrangiana para a primeira extensão proposta e, na Seção 5, é feita uma análise computacional deste método. Finalmente, na Seção 6, são apresentadas algumas conclusões e propostas para pesquisas futuras.

\section{Modelagem matemática}

\subsection{Modelo com itens diferentes}

A primeira extensão dos modelos de Norden e Velde (2005) e Molina et al. (2009) consiste em considerar que os itens a serem produzidos possuem 
tamanhos diferentes e não podem ser misturados em um mesmo palete. Ou seja, cada palete pode ser carregado com um único tipo de item, o que é usual na prática de produtores, especialmente em ambientes de manufatura. Observe que, para isso, é necessário definir a capacidade do palete para cada tipo de item. Este dado pode ser obtido a priori, resolvendo-se o chamado Problema de Carregamento de Paletes do Produtor (e.g., OLIVEIRA; MORABITO, 2006, MORABITO; PUREZA, 2009, BIRGIN; LOBATO; MORABITO, 2010) como um pré-processamento para cada tipo de item. Este problema de carregamento considera que os itens são arranjados em camadas horizontais sobre a superfície do palete, o que também é usual na prática dos produtores para facilitar o arranjo e a estabilidade da carga. Neste caso, tem-se que considerar esta restrição adicional no modelo de dimensionamento de lotes com custos de transporte. Os parâmetros e variáveis do modelo são descritos a seguir.

Parâmetros do modelo:

- $i=1,2, \ldots, n$ - número de itens distintos;

- $t=1,2, \ldots, T$ - número de períodos no horizonte de planejamento;

- $s_{i t}$ - custo de preparação para a produção do item $i$ no período $t$

- $h_{i t}^{+}$- custo unitário de estocagem do item $i$ no período $t$;

- $h_{i t}^{-}$- penalidade por atraso de uma unidade do item $i$ no período $t$;

- $\quad d_{i t}$ - demanda do item $i$ no período $t$;

- $b_{i}$ - tempo necessário para a produção de uma unidade do item $i$

- $q_{i}$ - tempo de preparação para a produção do item $i$;

- $C a p_{t}$ - capacidade de produção no período $t$;

- $\quad M$ - um número positivo suficientemente grande;

- $P_{i}$ - número de itens do tipo $i$ que podem ser colocados num mesmo palete (dado obtido resolvendo-se um problema de carregamento de palete para cada item $i$ );

- $c_{0}$ - custo fixo mensal do contrato;

- $c_{1}$ - custo unitário de transporte dos primeiros $R$ paletes utilizados;

- $c_{2}$ - custo unitário de transporte dos demais paletes $\left(c_{2} \gg c_{1}\right)$

- $R$ - número contratado de paletes com custo mais barato $c_{1}$.

Variáveis de decisão:

- $X_{i t}$ - quantidade a ser produzida do item $i$ no período $t$;

- $I_{i t}^{+}$- quantidade estocada do item $i$ no período $t$;

- $I_{i t}^{-}$- quantidade atrasada do item $i$ no período $t$;
- $Y_{i t}$ - variável binária que indica a produção do item $i$ no período $t\left(Y_{i t}=1\right.$ se $X_{i t}>0 ; Y_{i t}=0$, caso contrário);

- $A_{i t}$ - número de paletes transportados contendo o item $i$ no período $t$ com custo unitário $c_{1}$;

- $B_{i t}$ - número de paletes transportados contendo o item $i$ no período $t$ com custo unitário $c_{2}$.

A Figura 1 ilustra os custos de transporte lineares por partes e convexos em relação ao número de paletes utilizados. Observe que a partir da quantidade $R, \mathrm{o}$ custo unitário de transporte muda de $c_{1}$ para $c_{2}$.

$\mathrm{O}$ primeiro modelo estendido é definido por:

Modelo 1:

$$
\begin{aligned}
& \mathrm{z}=\min \sum_{\mathrm{i}=1}^{\mathrm{n}} \sum_{\mathrm{t}=1}^{\mathrm{T}}\left(h_{i t}^{+} I_{i t}^{+}+h_{i t}^{-} I_{i t}^{-}+s_{i t} Y_{i t}\right) \\
& +\sum_{t=1}^{T}\left(c_{0}+c_{1} \sum_{i=1}^{n} A_{i t}+c_{2} \sum_{i=1}^{n} B_{i t}\right)
\end{aligned}
$$

s.a. $I_{i, t-1}^{+}-I_{i, t-1}^{-}+X_{i t}-I_{i, t}^{+}+I_{i, t}^{-}=d_{i t}$

$i=1, \ldots, n ; t=1, \ldots, T$

$X_{i t}-M Y_{i t} \leq 0 \quad i=1, \ldots, n ; t=1, \ldots, T$

$$
\sum_{i=1}^{n}\left(b_{i} X_{i t}+q_{i} Y_{i t}\right) \leq \operatorname{Cap}_{t} \quad t=1, \ldots, T
$$

$$
B_{i t}+A_{i t} \geq \frac{X_{i t}}{P_{i}} \quad i=1, \ldots, n ; t=1, \ldots, T
$$

$$
0 \leq \sum_{i=1}^{n} A_{i t} \leq R \quad t=1, \ldots, T
$$

$$
I_{i 0}^{+}=I_{i 0}^{-}=I_{i T}^{-}=I_{i T}^{-}=0 \quad i=1, \ldots, n
$$

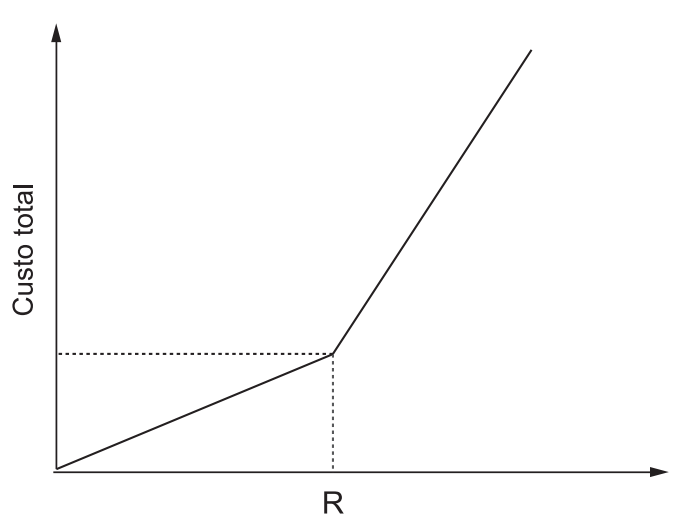

Quantidade de paletes

Figura 1. Custo convexo de transporte. 


$$
\begin{aligned}
& X_{i t} \geq 0, I_{i t}^{+} \geq 0, I_{i t}^{-} \geq 0 \quad i=1, \ldots, n ; t=1, \ldots, T \\
& A_{i t}, B_{i t} \in Z^{+}, Y_{i t} \in\{0,1\} \quad i=1, \ldots, n ; t=1, \ldots, T
\end{aligned}
$$

As diferenças entre o Modelo 1 e o modelo tratado em Molina et al. (2009) estão na definição do parâmetro $P_{i}$ e das variáveis $A_{i t}$ e $B_{i t}$ e, portanto, na Função Objetivo 1 e Restrições 1, 5, 6 e 9. A função objetivo (1) minimiza a soma dos custos de estoque, de atraso, de preparação e de transporte. As restrições (2) garantem que a demanda é atendida. As restrições (3) asseguram que exista produção apenas quando o custo e o tempo de preparação forem considerados. As restrições (4) são restrições de capacidade de produção. Nas restrições (5), é assegurado que um número suficiente de paletes para o transporte de cada tipo de item é alocado. As restrições (6) limitam em $R$ a utilização dos paletes com custo mais baixo. Em (7) é imposto que os estoques e atrasos iniciais e finais dos itens sejam nulos, e as restrições (8) garantem a não negatividade das variáveis de produção, de estoque e de atraso. Finalmente, em (9) são definidos os domínios das variáveis binárias e inteiras. Para este modelo, $M$ é fixado como a soma das demandas de todos os itens em todo o horizonte de planejamento, $M=\sum_{i=1}^{n} \sum_{t=1}^{T} d_{i t}$.

\subsection{Modelo considerando o transporte dos paletes em caminhões iguais}

A segunda extensão do problema de dimensionamento de lotes com custos de transporte considera que os itens a serem produzidos possuem tamanhos diferentes e não podem ser misturados em um mesmo palete (como no Modelo 1) e, além disso, depois de alocar os itens nos paletes, estes últimos são carregados em caminhões de mesmo tamanho. Os custos de transporte não são mais diretamente associados ao número de paletes utilizados, e sim ao número de caminhões necessários para fazer o transporte dos paletes. Observe que, além do problema de empacotamento de itens (de tamanhos iguais) no palete (um problema para cada tamanho de item), tem-se o problema de empacotamento de paletes (de tamanhos iguais) no caminhão. Ambos os problemas de empacotamento podem ser considerados num pré-processamento, resolvendo-se problemas de carregamento de paletes do produtor e admitindo-se que os paletes são arranjados em camadas horizontais dentro da carroceria do caminhão, o que é usual na prática.

Parâmetros adicionais do modelo:

- $P^{\prime}$ - número máximo de paletes que pode ser transportado em um caminhão (dado obtido resolvendo-se um problema de carregamento de palete correspondente, em que os itens do problema são os paletes e o palete do problema é o caminhão);

- $c_{1}$ - custo unitário dos primeiros $R$ caminhões utilizados;

- $c_{2}$ - custo unitário dos demais caminhões $\left(c_{2} \gg c_{1}\right)$;

- $R^{\prime}$ - número contratado de caminhões com custo mais barato $c_{1}$.

Variáveis de decisão adicionais:

- $A_{t}$ - número de caminhões utilizados no período $t$ com custo unitário $c_{1}$;

- $B_{t}$ - número de caminhões utilizados no período $t$ com custo unitário $\mathrm{c}_{2}$;

- $Z_{i t}$ - número de paletes necessários para transportar os itens do tipo $i$ produzidos no período $t$.

O segundo modelo estendido é dado por:

Modelo 2:

$$
\begin{aligned}
& \mathrm{z}=\min \sum_{i=1}^{n} \sum_{t=1}^{T}\left(h_{i t}^{+} I_{i t}^{+}+h_{i t}^{-} I_{i t}^{-}+s_{i t} Y_{i t}\right) \\
& +\sum_{t=1}^{T}\left(c_{0}+c_{1} A_{t}+c_{2} B_{t}\right)
\end{aligned}
$$

s.a. (Equações 2, 3, 4, 7, 8)

$$
\begin{gathered}
B_{t}+A_{t} \geq \frac{1}{P^{\prime}} \sum_{i=1}^{n} Z_{i t} \quad t=1, \ldots, T \\
Z_{i t} \geq \frac{X_{i t}}{P_{i}} \quad i=1, \ldots, n ; t=1, \ldots, T \\
0 \leq A_{t} \leq R^{\prime} \quad t=1, \ldots, T \\
A_{t}, B_{t}, Z_{i t} \in Z^{+}, Y_{i t} \in\{0,1\} \\
i=1, \ldots, n ; t=1, \ldots, T
\end{gathered}
$$

A função objetivo (10) e as restrições (13 e 14) foram simplesmente adaptadas, de (1, 6 e 9), respectivamente, para o problema em questão. As restrições (11) determinam o número de caminhões necessários para transportar os paletes pelo custo mais barato e pelo custo mais caro, e as restrições (12) referem-se ao número de paletes necessários para o transporte de cada tipo de item em cada período. As restrições (13) limitam o número de caminhões mais baratos e (14) definem o domínio das variáveis. 


\subsection{Modelo considerando o transporte dos paletes em caminhões diferentes}

A terceira extensão do modelo de dimensionamento de lotes com custos de transporte considera, além das extensões dos modelos das Seções 2.1 e 2.2, a existência de vários tipos de caminhões com capacidades e custos diferentes, o que ocorre na prática em situações de frota heterogênea. Similarmente aos casos das Seções 2.1 e 2.2, o problema de empacotamento relativo ao carregamento de paletes (de tamanhos iguais) em cada caminhão pode ser resolvido num pré-processamento, por meio da solução de problemas de carregamento de paletes do produtor (um problema para cada tipo de caminhão).

Observe que o modelo a seguir envolve decidir quantos caminhões de cada tipo utilizar, o que o diferencia dos modelos anteriores, em que se determina apenas uma quantidade, seja de paletes ou de caminhões, sem a necessidade de se escolher entre diferentes opções de paletes ou caminhões com diferentes custos. Portanto, a decisão agora é relativa ao custo de cada caminhão. Caminhões menores têm custos unitários menores, mas têm capacidade restrita a um menor número de paletes. Considere os seguintes dados e variáveis adicionais aos dados dos Modelos 1 e 2:

Parâmetros adicionais do modelo:

- $k=1, \ldots, K$ - Número de tipos de caminhões distintos;

- $P_{k}^{\prime}$ - número máximo de paletes que pode ser transportado no caminhão do tipo $k$ (dado obtido resolvendo-se um problema de carregamento de palete correspondente, em que os itens do problema são os paletes e o palete do problema é o caminhão do tipo $k$ );

- $c_{1 k}$ - custo unitário dos primeiros $R$ caminhões do tipo $k$ utilizados;

- $c_{2 k}$ - custo unitário dos demais caminhões do tipo $k\left(c_{2 k} \gg c_{1 k}\right)$;

- $R_{k}^{\prime}$ - número contratado de caminhões do tipo $k$ com custo mais barato $c_{1 k}$.

Variáveis de decisão adicionais:

- $A_{k t}$ - número de caminhões do tipo $k$ utilizados no período $t$ com custo unitário $c_{1 k}$;

- $B_{k t}$ - número de caminhões do tipo $k$ utilizados no período $t$ com custo unitário $c_{2 k}$;

- $Z_{k t}^{\prime}$ - número de caminhões do tipo $k$ para o transporte de paletes no período $t$.

O modelo estendido é dado por:

Modelo 3:

$$
\begin{aligned}
& \mathrm{z}=\min \sum_{i=1}^{n} \sum_{t=1}^{T}\left(h_{i t}^{+} I_{i t}^{+}+h_{i t}^{-} I_{i t}^{-}+s_{i t} Y_{i t}\right)+ \\
& \sum_{t=1}^{T}\left(c_{0}+\sum_{k=1}^{K}\left(c_{1 k} A_{k t}+c_{2 k} B_{k t}\right)\right)
\end{aligned}
$$

s.a. (Equações 2, 3, 4, 7, 8, 12).

$$
\begin{gathered}
A_{k t}+B_{k t} \geq \frac{Z_{k t}^{\prime}}{P_{k}} \quad t=1, \ldots, T ; k=1, \ldots, K \\
\sum_{k=1}^{K} Z_{k t}^{\prime}=\sum_{i=1}^{n} Z_{i t} \quad t=1, \ldots, T \\
0 \leq A_{k t} \leq R_{k}^{\prime} \quad t=1, \ldots, T ; k=1, \ldots, K \\
A_{k t}, B_{k t}, Z_{i t}, Z_{k t}^{\prime} \in Z^{+}, Y_{i t} \in\{0,1\} \\
i=1, \ldots, n ; t=1, \ldots, T ; k=1, \ldots, K
\end{gathered}
$$

A função objetivo (15) foi modificada em relação à (1) para retratar os novos custos de transporte. A restrição (16) calcula o número de caminhões necessário para o transporte dos paletes no período $t$. A restrição (17) garante que o número de paletes transportado pelos caminhões seja igual ao número de paletes necessário para transportar a produção de cada período. Note que o modelo não tem a informação de qual item está sendo carregado por qual tipo de caminhão, mas esta informação não é relevante, dado que estamos considerando que os paletes têm tamanhos iguais e os itens têm o mesmo destino (i.e., o depósito). As restrições (18) limitam a utilização dos caminhões do tipo $k$ com custo mais baixo $R_{k}^{\prime}$. As restrições (19) definem o domínio das variáveis

O Modelo 3 pode ser particularizado para o caso em que se tem apenas um tipo de custo contratual (apenas os valores de $c_{1 k}$ ) para cada tipo de caminhão $k$. Neste caso, as decisões envolvem apenas a escolha dos caminhões de cada tipo, que, por sua vez, estão disponíveis em quantidades limitadas. Observe que o problema pode se tornar infactível caso o número de caminhões não seja suficiente para o transporte de toda a produção. Esta modificação do Modelo 3 também pode ser vista como o caso em que o custo $c_{2 k}$ é suficientemente grande. Para os testes computacionais da próxima seção, o Modelo 3 modificado foi utilizado.

\section{Análise computacional dos modelos}

Nesta seção, inicialmente é descrita a maneira como os dados dos experimentos foram gerados e, posteriormente, apresentam-se alguns resultados computacionais. Os Modelos 1, 2 e 3 foram codificados na linguagem de modelagem AMPL e resolvidos pelo pacote computacional CPLEX 10.0 com parâmetros default, utilizando um microcomputador Pentium Core 2 Duo 2GHZ com $2 \mathrm{G}$ de memória RAM e sistema operacional Windows XP. 
Tabela 1. Dados fixos dos modelos.

\begin{tabular}{|c|c|}
\hline$s_{i t}$ & 50 \\
\hline$h_{i t}^{+}$ & 3 \\
\hline$h_{i t}^{-}$ & 10 \\
\hline$b_{i}$ & 1 \\
\hline$q_{i}$ & {$[10,30]$} \\
\hline$E D_{i}$ & {$[40,700]$} \\
\hline$d_{i t}$ & $\left.\left[\frac{E D_{i}}{2}\right\rfloor,\left[\frac{3 E D_{i}}{2}\right\rceil\right]$ \\
\hline$P_{i}$ & {$[50,150]$} \\
\hline$F R$ & $\frac{1}{4}$ \\
\hline
\end{tabular}

\subsection{Geração dos dados e testes iniciais}

Para as classes do Modelo 1, são considerados os seguintes dados baseados em Norden e Velde (2005) e Molina et al. (2009). A Tabela 1 apresenta dois tipos de dados fixos, valores constantes e intervalos. Quando os valores forem representados por um intervalo, significa que o valor deste dado foi escolhido de modo aleatório e uniforme dentro deste intervalo. No processo de geração dos dados, têm-se picos de demanda a cada cinco períodos, em que a demanda $d_{i t}$ é multiplicada por 1,5 . A capacidade de produção em cada período é gerada de forma dependente dos tempos de produção e preparação e da demanda de cada item pela Equação 20:

$$
\operatorname{Cap}_{t}=\left\lfloor\frac{\sum_{i=1}^{n} \sum_{j=1}^{T}\left(b_{i} d_{i j}+q_{i}\right)}{\alpha T}\right\rfloor t=1, \ldots, T
$$

em que $\alpha$ é um parâmetro para controle da folga na capacidade. O número máximo de paletes baratos, ou seja, o valor $R$ é calculado de acordo com a fração de demanda média que é transportada com o custo $c_{1}$. O valor de $F R$ significa que $R$ deve ser gerado de forma que, para uma fração $F R$ dos períodos (por exemplo, $F R=1 / 4$ ), a demanda deve ser inferior a $R\left(\sum_{i=1}^{n} P_{i} / n\right)$. Na adaptação deste valor para o Modelo 2, considera-se o número de

paletes que pode ser transportado no caminhão $\left(P^{\prime}\right)$, ou seja, $R$ ' deve ser gerado de forma que, para uma fração $F R=1 / 4$ dos períodos, a demanda deve ser inferior a $R^{\prime}\left[\left(\left(\sum_{i=1}^{n} P_{i} / n\right) / P^{\prime}\right)\right]$. Como o Modelo 3 considera caminhões de tamanhos diferentes e os caminhões menores são limitados em $R$, neste caso
$F R$ é interpretado como a fração de períodos em que a demanda é inferior a $R^{\prime}\left[\left(\sum_{i=1}^{n} P_{i}\right) / P_{k}^{\prime}\right]$.

Além desses dados, há quatro outros dados que definem as classes de exemplares : número de itens $(n)$; número de períodos $(T)$; custos de transporte $\left(c_{1} \mathrm{e} c_{2}\right)$; e o controle de folga na capacidade $(\alpha)$. Baseando-se em Molina et al. (2009), inicialmente foram geradas quatro classes de exemplares para os problemas de tamanhos moderados (classes M1, M2, M3 e M4), para testar as extensões do modelo. Foram considerados $n=20$ tipos de itens; $T=12$ períodos; os custos de transporte $\left(c_{1}, c_{2}\right)$ iguais a $(50,200)$ e $(150,500)$; e o controle de folga na capacidade $(\alpha)$ igual a 0,85 e 1 . Para cada uma dessas quatro classes, foram gerados 50 exemplares que diferem entre si apenas pelos dados que são gerados aleatoriamente nos intervalos predeterminados. Convém observar que estes exemplares de tamanhos moderados já representam situações realistas na prática, conforme Norden e Velde (2005) e Molina et al. (2009).

Em seguida, para uma melhor análise do comportamento e das limitações dos Modelos 1, 2 e 3, foram geradas outras 16 classes do problema com exemplares de tamanhos grandes (classes G1, G2, ..., G16), com números de itens iguais a 50 e 150, e números de períodos iguais a 20 e 50 . Novamente, para cada uma destas dezesseis classes, foram gerados 50 exemplares que diferem entre si apenas pelos dados que são gerados aleatoriamente nos intervalos predeterminados. A Tabela 2 apresenta as dimensões das classes de exemplares M1-M4 e G1-G16.

Além dos dados gerados para o Modelo 1, no Modelo 2, $P$ ' foi gerado uniformemente dentro do intervalo $[40,80]$ (i.e., $P^{\prime} \in[40,80]$ ). O restante dos dados foi mantido igual aos dados do Modelo 1. Observe que, agora, os custos são referentes ao número de caminhões. No Modelo 3, como mencionado anteriormente, foi considerada uma particularização do Modelo 1, em que os custos pela utilização dos caminhões extras são suficientemente grandes. Assim, o modelo fica equivalente ao caso em que se tem que decidir apenas a quantidade e os tipos de caminhões que serão utilizados, ou seja, sem a decisão de utilizar ou não os caminhões extras. Considere os seguintes dados adicionais para o Modelo 3: número de caminhões $K=2, P_{1}^{\prime} \in[40,80], P_{2}^{\prime}=2 \times P_{1}^{\prime}, c_{1}=50$ e 150 e $c_{2}=1,5 \times c_{1}$.

\subsection{Resultados computacionais dos exemplares de tamanho moderado (classes M1-M4)}

Os resultados computacionais obtidos com o pacote AMPL/CPLEX para as classes M1-M4 são apresentados nas Tabelas 3-5 e foram analisados considerando-se: 
- NC: número médio dos nós com a melhor solução;

- $N N$ : número médio de nós do CPLEX;

- $P C$ : número médio de planos de corte do CPLEX;

- TC: tempo computacional médio do CPLEX (limitado em 180 segundos);

- $G A P$ : valor médio de $G A P=|U B-L B| \mid$ $\left(\mathrm{e}^{-10}+|U B|\right)$ para os 50 exemplares, em que $U B$ e $L B$ são os limitantes superior e inferior do CPLEX.

Os planos de corte $(P C)$ são detalhados a seguir, pois, em Molina (2007), foram feitos testes com o pacote AMPL/CPLEX em problemas de dimensionamento de lotes com custos de transporte, e o autor concluiu que a geração de planos de corte é a uma ferramenta importante para obter boas soluções

Tabela 2. Dados variáveis do Modelo 1.

\begin{tabular}{lrccrc}
\hline & $\begin{array}{c}\text { Item } \\
(\boldsymbol{n})\end{array}$ & $\begin{array}{c}\text { Período } \\
(\boldsymbol{T})\end{array}$ & $\alpha$ & $\boldsymbol{c}_{\mathbf{1}}$ & $\boldsymbol{c}_{\mathbf{2}}$ \\
\hline classe M1 & 20 & 12 & 0,85 & 50 & 200 \\
classe M2 & 20 & 12 & 0,85 & 150 & 500 \\
classe M3 & 20 & 12 & 1,00 & 50 & 200 \\
classe M4 & 20 & 12 & 1,00 & 150 & 500 \\
classe G1 & 50 & 20 & 0,85 & 50 & 200 \\
classe G2 & 50 & 50 & 0,85 & 50 & 200 \\
classe G3 & 150 & 20 & 0,85 & 50 & 200 \\
classe G4 & 150 & 50 & 0,85 & 50 & 200 \\
classe G5 & 50 & 20 & 1,00 & 50 & 200 \\
classe G6 & 50 & 50 & 1,00 & 50 & 200 \\
classe G7 & 150 & 20 & 1,00 & 50 & 200 \\
classe G8 & 150 & 50 & 1,00 & 50 & 200 \\
classe G9 & 50 & 20 & 0,85 & 150 & 500 \\
classe G10 & 50 & 50 & 0,85 & 150 & 500 \\
classe G11 & 150 & 20 & 0,85 & 150 & 500 \\
classe G12 & 150 & 50 & 0,85 & 150 & 500 \\
classe G13 & 50 & 20 & 1,00 & 150 & 500 \\
classe G14 & 50 & 50 & 1,00 & 150 & 500 \\
classe G15 & 150 & 20 & 1,00 & 150 & 500 \\
classe G16 & 150 & 50 & 1,00 & 150 & 500 \\
\hline
\end{tabular}

para estes problemas. Nos resultados da Tabela 3, considera-se o Modelo 1 e pode-se observar que em todas as classes o pacote de otimização utiliza o tempo máximo de resolução e, embora obtenha um GAP pequeno, não prova a otimalidade da solução encontrada. Nestes testes não foi possível identificar porque a classe M4 difere das outras na geração dos planos de corte; porém observe que nos exemplares desta classe o pacote analisa um número muito maior de nós na árvore de busca do método de resolução.

De acordo com os resultados da Tabela 4, nos testes realizados com o Modelo 2, o pacote de otimização encontra a solução ótima de todos os problemas das classes M1-M4, diferentemente dos resultados dos problemas do Modelo 1, em que o pacote de otimização encontra apenas uma solução viável sem conseguir provar sua otimalidade.

Considerando os resultados para o Modelo 3 (Tabela 5), o pacote encontra a solução ótima para a maior parte dos exemplares. Apenas em alguns exemplares das classes M2 e M3 o pacote não prova a otimalidade da solução no tempo estipulado.

Pode-se concluir assim que os Modelos 1, 2 e 3, que representam o problema de dimensionamento de lotes com custo de transporte para casos realistas, podem ser razoavelmente bem resolvidos pelo pacote AMPL/ CPLEX em um tempo aceitável, considerando as decisões práticas (3 minutos).

\subsection{Resultados computacionais dos exemplares de tamanho grande (classes G1-G16)}

Na Tabela 6, são apresentadas as médias dos resultados obtidos para os $16 \times 50=800$ exemplares das classes G1-G16 para o Modelo 1. Deve-se observar que o Modelo 1 possui $(3 n T)$ variáveis reais, $(2 n T)$ inteiras e $(n T)$ variáveis binárias. Além disso, o modelo possui $T(3 n+2)+4 n$ restrições. Por exemplo, na classe G16 os modelos têm 22.500 variáveis reais, 15.000 variáveis inteiras, 7.500 variáveis binárias e 23.200 restrições (enquanto que na classe M4 da Tabela 1 os modelos têm apenas 720 variáveis reais, 480 variáveis inteiras, 240 variáveis binárias e 824 restrições). Em todos os exemplares resolvidos, o pacote AMPL/CPLEX atingiu o limite de tempo

Tabela 3. Resultados obtidos pelo AMPL/CPLEX-Modelo 1.

\begin{tabular}{|c|c|c|c|c|c|c|c|c|}
\hline & \multirow[b]{2}{*}{$N C$} & \multirow[b]{2}{*}{$N N$} & \multicolumn{4}{|c|}{$P C$} & \multirow[b]{2}{*}{$T C$} & \multirow{2}{*}{$\begin{array}{l}G A P \\
(\%)\end{array}$} \\
\hline & & & $\begin{array}{c}\text { Corte de } \\
\text { fluxo }\end{array}$ & $\begin{array}{c}\text { Inteiro } \\
\text { misto }\end{array}$ & $\begin{array}{c}\text { Corte de caminho } \\
\text { de fluxo }\end{array}$ & $\begin{array}{l}\text { Corte de } \\
\text { Gomory }\end{array}$ & & \\
\hline classe M1 & 72911,53 & 153361,59 & 101,16 & 233,49 & 49,71 & 241,47 & 180,01 & 0,01 \\
\hline classe M2 & 42223,61 & 69433,24 & 108,24 & 179,55 & 72,86 & 222,02 & 180,01 & 0,01 \\
\hline classe M3 & 42228,31 & 67416,59 & 99,00 & 184,10 & 77,37 & 229,14 & 180,01 & 0,01 \\
\hline classe M4 & 139262,67 & 174115,24 & 59,14 & 190,78 & 45,78 & 241,84 & 180,01 & 0,01 \\
\hline
\end{tabular}


Tabela 4. Resultados obtidos pelo AMPL/CPLEX-Modelo 2.

\begin{tabular}{rrrrccrrrr}
\hline & \multirow{2}{*}{$\boldsymbol{N C}$} & \multirow{N}{*}{$\boldsymbol{N}$} & $\begin{array}{c}\text { Corte de } \\
\text { fluxo }\end{array}$ & $\begin{array}{c}\text { Inteiro } \\
\text { misto }\end{array}$ & $\begin{array}{c}\text { Corte de caminho } \\
\text { de fluxo }\end{array}$ & $\begin{array}{c}\text { Corte de } \\
\text { Gomory }\end{array}$ & $\boldsymbol{T C}$ & $\begin{array}{c}\text { GAP } \\
(\boldsymbol{\%})\end{array}$ \\
\hline classe M1 & 13330,16 & 40773,96 & 246,84 & 214,10 & 22,80 & 97,80 & 22,13 & 0,00 \\
classe M2 & 55804,61 & 119805,86 & 276,82 & 195,04 & 57,86 & 91,59 & 122,30 & 0,00 \\
classe M3 & 65563,33 & 133345,43 & 280,76 & 192,92 & 57,90 & 85,61 & 131,77 & 0,00 \\
classe M4 & 55157,12 & 138227,35 & 253,73 & 221,08 & 25,10 & 100,82 & 70,52 & 0,00 \\
\hline
\end{tabular}

Tabela 5. Resultados obtidos pelo AMPL/CPLEX-Modelo 3.

\begin{tabular}{lrrrcccccc}
\hline & \multirow{2}{*}{$\boldsymbol{N C}$} & \multirow{N}{*}{$\boldsymbol{N}$} & & $\begin{array}{c}\text { Corte de } \\
\text { fluxo }\end{array}$ & $\begin{array}{c}\text { Inteiro } \\
\text { misto }\end{array}$ & $\begin{array}{c}\text { Corte de caminho } \\
\text { de fluxo }\end{array}$ & $\begin{array}{c}\text { Corte de } \\
\text { Gomory }\end{array}$ & TC & $\begin{array}{c}\text { GAP } \\
(\%)\end{array}$ \\
\hline classe M1 & 8477,55 & 25253,31 & 245,02 & 222,80 & 17,04 & 120,31 & 16,08 & 0,00 \\
classe M2 & 67255,98 & 189950,45 & 268,51 & 204,12 & 56,65 & 129,29 & 172,16 & 0,01 \\
classe M3 & 72979,92 & 160808,96 & 259,61 & 210,88 & 60,65 & 150,02 & 175,75 & 0,01 \\
classe M4 & 19893,78 & 87496,65 & 238,71 & 238,35 & 20,76 & 137,59 & 55,97 & 0,00 \\
\hline
\end{tabular}

Tabela 6. Resultados obtidos pelo AMPL/CPLEX-Modelo 1.

\begin{tabular}{|c|c|c|c|c|c|c|c|c|}
\hline & \multirow[b]{2}{*}{$N C$} & \multirow[b]{2}{*}{$N N$} & \multicolumn{4}{|c|}{$P C$} & \multirow[b]{2}{*}{$T C$} & \multirow{2}{*}{$\begin{array}{l}\text { GAP } \\
(\%)\end{array}$} \\
\hline & & & $\begin{array}{l}\text { Corte de } \\
\text { fluxo }\end{array}$ & $\begin{array}{l}\text { Inteiro } \\
\text { misto }\end{array}$ & $\begin{array}{c}\text { Corte de caminho } \\
\text { de fluxo }\end{array}$ & $\begin{array}{l}\text { Corte de } \\
\text { Gomory }\end{array}$ & & \\
\hline classe G1 & 6393,00 & 31119,93 & 1396,23 & 1316,60 & 102,67 & 399,73 & 180,00 & 1,69 \\
\hline classe G2 & 8013,00 & 8995,97 & 4123,37 & 3200,27 & 268,67 & 400,00 & 180,00 & 1,91 \\
\hline classe G3 & 6838,67 & 7925,30 & 5081,40 & 3902,43 & 312,93 & 400,00 & 180,00 & 1,66 \\
\hline classe G4* & 642,27 & 3309,90 & 13742,00 & 9566,70 & 832,20 & 400,00 & 180,00 & 3,66 \\
\hline classe G5 & 4744,00 & 21344,73 & 1381,70 & 956,00 & 352,37 & 392,57 & 180,00 & 3,83 \\
\hline classe G6 & 8219,64 & 8925,68 & 3900,60 & 2006,83 & 1050,43 & 396,23 & 180,00 & 6,15 \\
\hline classe G7** & 2735,00 & 3546,00 & 4825,90 & 2741,03 & 1323,03 & 398,60 & 180,00 & 7,25 \\
\hline classe G8 & - & 5,33 & 13280,30 & 6173,67 & 3089,37 & 399,90 & 180,00 & - \\
\hline classe G9** & 4935,13 & 29602,20 & 1337,93 & 1204,17 & 144,33 & 399,70 & 180,00 & 2,92 \\
\hline classe G10 & 9971,79 & 10846,10 & 3998,17 & 2881,00 & 373,23 & 399,97 & 180,00 & 3,47 \\
\hline classe G11*** & 7415,00 & 9247,03 & 4934,50 & 3556,00 & 432,33 & 399,93 & 180,00 & 6,56 \\
\hline classe G12 & - & 588,77 & 13365,53 & 8731,50 & 1183,10 & 399,93 & 180,00 & - \\
\hline classe G13 & 4643,33 & 12928,83 & 1361,93 & 947,47 & 347,13 & 391,77 & 180,00 & 4,34 \\
\hline classe G14 & - & 6114,20 & 3835,43 & 1893,70 & 1064,23 & 396,00 & 180,00 & - \\
\hline classe G15 & - & 2377,57 & 4786,40 & 2679,23 & 1318,23 & 398,23 & 180,00 & - \\
\hline classe G16 & - & 4,33 & 13121,97 & 5355,20 & 3088,73 & 399,83 & 180,00 & - \\
\hline
\end{tabular}

*Não foi possível encontrar uma solução factível para 9 exemplares, os quais foram retirados para a obtenção da média. **Não foi possível encontrar uma solução factível para 2 exemplares, os quais foram retirados para a obtenção da média. ***Não foi possível encontrar uma solução factível para 48 exemplares, os quais foram retirados para a obtenção da média. -Não foi possível encontrar uma solução factível para os 50 exemplares.

antes de encontrar uma solução ótima e provar sua otimalidade.

Podemos verificar que há um compromisso ou compensação (trade-off) entre resolver a árvore de decisão e gerar planos de corte. Isto pode ser visto verificando que quanto maior o número de planos de corte gerados, menor é o número de nós avaliados. O pacote obtém boas soluções iniciais com a geração de planos de corte, não passando de $10 \%$ de GAP já na primeira solução (nó raiz) dos exemplares em que obteve uma solução factível dentro do limite de tempo. Quanto maior o tamanho do problema (representado pelo número de itens e períodos), maior a dificuldade em obter boas soluções (veja 
Tabela 7. Resultados obtidos pelo AMPL/CPLEX - Modelo 2.

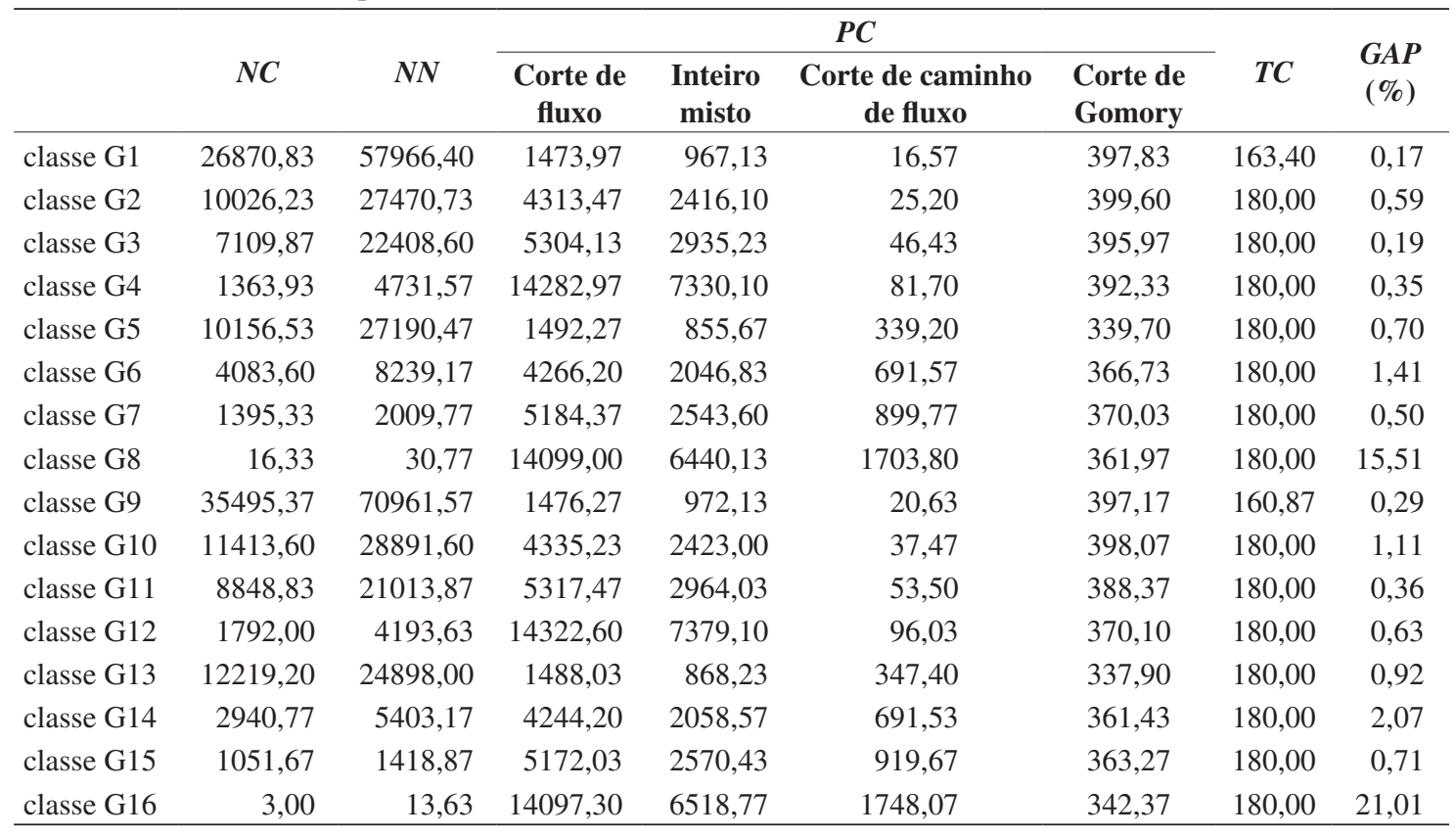

coluna GAP). Além disso, o aumento nos custos de transporte também dificulta bastante a resolução do problema, provavelmente devido a um trade-off maior entre os custos de preparação e transporte.

Na Tabela 7, são apresentados os resultados médios obtidos com o Modelo 2 para os respectivos 800 exemplares das classes G1-G16. O Modelo 2 possui $2 T(n+1)$ variáveis inteiras e o restante das variáveis se mantém com o mesmo número do Modelo 1. Além disso, o Modelo 2 tem $T(3 n T+3)+4 n$ restrições. Por exemplo, na classe G16 os modelos têm 22.500 variáveis reais, 100 variáveis inteiras, 7.500 variáveis binárias e 68.550 restrições (enquanto que na classe M4 da Tabela 2, os modelos têm apenas 720 variáveis reais, 24 variáveis inteiras, 240 variáveis binárias e 836 restrições). É possível observar que para o Modelo 2 o pacote teve menos dificuldades para resolver estes exemplares do que para o Modelo 1. Nas classes G1 e G9, o pacote prova a otimalidade da solução encontrada para alguns exemplares (observe que o tempo médio é menor nestas classes). O pacote encontra pelo menos uma solução factível para todos os 800 exemplares gerados. Novamente, nota-se que quando se tem um maior número de itens e períodos, o pacote tem mais dificuldades para resolver o problema (classes G8 e G16) e é gerado um maior número de planos de corte nestas classes. Outra observação nesta tabela é em relação à dificuldade maior para resolver exemplares com altos custos de transporte. Note na classe G16 que a melhor solução foi encontrada no terceiro nó da árvore de busca, com GAP de 21\%, e que foram analisados em média 14 nós em cada problema.

Finalmente, a Tabela 8 apresenta os resultados obtidos com o Modelo 3 para os respectivos 800 exemplares das classes G1-G16. O Modelo 3 contém $(n T+3 n K)$ variáveis inteiras e o restante se mantém com o mesmo número dos outros modelos. Em relação ao número de restrições, o Modelo 3 possui $(3 n T+2 T+4 n+2 K T)$ restrições. Por exemplo, na classe G16 os modelos têm 22.500 variáveis reais, 7.800 variáveis inteiras, 7.500 variáveis binárias e 23.400 restrições (enquanto que na classe M4 da Tabela 3, os modelos têm apenas 720 variáveis reais, 312 variáveis inteiras, 240 variáveis binárias e 872 restrições). Pode-se observar na Tabela 8 que o pacote AMPL/CPLEX também teve mais dificuldades para resolver alguns exemplares do Modelo 3, particularmente as das classes G8 e G16 (exemplares com maior número de variáveis). Em todos os exemplares, o pacote utilizou o limite de tempo, e para nenhum exemplar foi provada a otimalidade da solução. Além disso, o número de planos de corte gerados segue a mesma tendência a cada quatro classes, de acordo com o aumento no tamanho do problema.

A partir da análise dos resultados apresentados nas três tabelas anteriores, é possível concluir que o pacote de otimização é razoavelmente eficaz na resolução dos Modelos 1, 2 e 3 de dimensionamento de lotes com custos de transporte propostos. Entretanto, considerando o limite de tempo de 180 segundos, para algumas classes o gap ainda é relativamente alto e, particularmente para o Modelo 1, o pacote teve dificuldades em encontrar 
Tabela 8. Resultados obtidos pelo AMPL/CPLEX - Modelo 3.

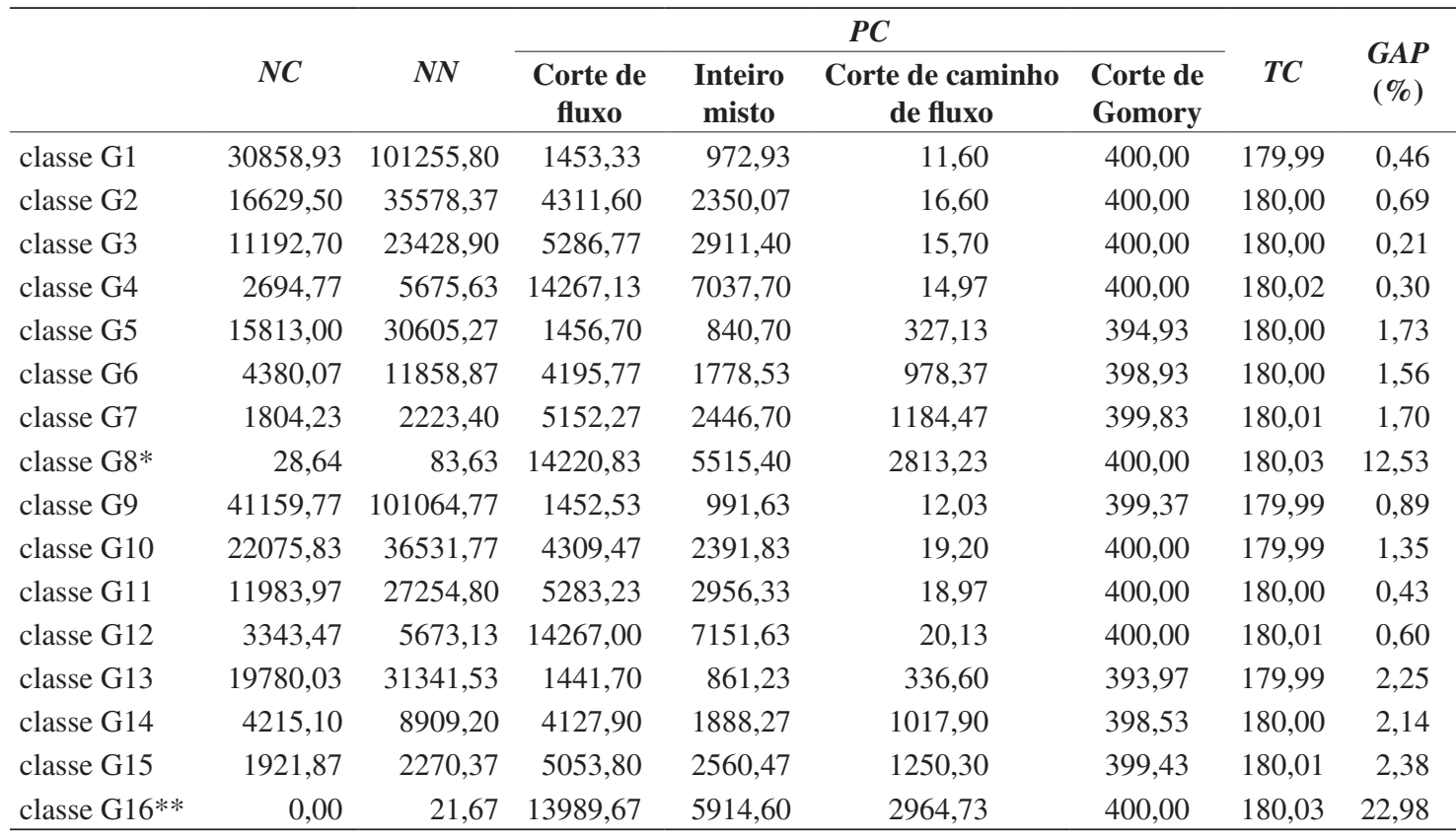

*Não foi possível encontrar uma solução factível para 9 exemplares, os quais foram retirados para a obtenção da média. **Não foi possível encontrar uma solução factível para 49 exemplares, os quais foram retirados para a obtenção da média.

soluções factíveis. Diante destes resultados, apresentamos a seguir alguns resultados computacionais adicionais em que aumentamos o limite de tempo para 1500 segundos, que ainda pode ser considerado viável na prática quando se trata de decisões de nível tático. Além disso, como uma alternativa ao pacote comercial, adaptamos a heurística Lagrangiana, proposta em Molina et al. (2009) para o problema mais simples apresentado em Norden e Velde (2005), para resolver o problema do Modelo 1. Uma descrição resumida desta heurística é feita na próxima seção, e são apresentados alguns resultados computacionais comparando o desempenho da heurística com aqueles obtidos com o pacote de otimização.

\section{Método heurístico de resolução para o modelo com itens diferentes (Modelo 1)}

A heurística Lagrangiana proposta em Molina et al. (2009) pode ser adaptada para a resolução do problema de dimensionamento de lotes com custos de transporte apresentado na Seção 2.1 (Modelo 1). Os passos da heurística proposta são aqui apresentados de forma resumida; mais detalhes podem ser encontrados em Molina (2011). A ideia básica da heurística consiste em relaxar as restrições de capacidade (4) e transporte (5) e penalizá-las na função objetivo (1). Assim, como acontece em Molina et al. (2009), o problema relaxado pode ser separado em dois subproblemas independentes, um de dimensionamento de lotes e outro de transporte dos paletes:

- Subproblema de dimensionamento de lotes

$$
\begin{aligned}
& z_{1}=\min \sum_{i=1}^{n} \sum_{\mathrm{t}=1}^{T}\left(\begin{array}{l}
h_{i t}^{+} I_{i t}^{+}+h_{i t}^{-} I_{i t}^{-}+\left(s_{i t}+\mu_{t}^{1} q_{i}\right) Y_{i t} \\
+\left(\mu_{t}^{1} b_{i}+\frac{\mu_{i t}^{2}}{P_{i}}\right) X_{i t}
\end{array}\right)(21) \\
& -\sum_{t=1}^{T} \mu_{t}^{1} \operatorname{Cap}_{t}
\end{aligned}
$$

s.a (Equações 2, 3, 7, 8).

$$
Y_{i t} \in\{0,1\} \quad i=1, \ldots, n ; t=1, \ldots, T
$$

em que $\mu_{t}^{1}$ denota o multiplicador lagrangiano relacionado à restrição de capacidade e $\mu_{i t}^{2} \mathrm{o}$ multiplicador lagrangiano relacionado à restrição de transporte dos paletes. O subproblema $\left(z_{1}\right)$, dado pela função objetivo (21) e restrições $(2,3,7,8$ e 22), pode ser decomposto em $n$ problemas de dimensionamento de lotes, um para cada item. A solução ótima para cada item pode ser facilmente obtida utilizando-se o algoritmo de Zangwill (1969).

- Subproblema de transporte dos itens

$$
\begin{aligned}
& z_{2}=\min \sum_{t=1}^{T}\left(c_{0}+c_{1} \sum_{i=1}^{n} A_{i t}+c_{2} \sum_{i=1}^{n} B_{i t}\right) \\
& +\sum_{i=1}^{n} \sum_{t=1}^{T} \mu_{i t}^{2}\left(-A_{i t}-B_{i t}\right)
\end{aligned}
$$


s.a. (Inequação 6)

$$
A_{i t}, B_{i t} \in Z^{+} \quad i=1, \ldots, n ; t=1, \ldots, T
$$

Como o custo $\sum_{t=1}^{T} c_{0}$ é constante e independe da decisão tomada, este pode ser omitido. Neste trabalho, o custo de contrato é considerado como nulo. Assim, a função objetivo (23) pode ser rescrita por:

$$
z_{2}=\min \sum_{t=1}^{T} \sum_{i=1}^{n}\left(\left(c_{1}-\mu_{i t}^{2}\right) A_{i t}+\left(c_{2}-\mu_{i t}^{2}\right) B_{i t}\right)(25)
$$

A resolução de $\left(z_{2}\right)$, dado pela função objetivo (25) e restrições ( 6 e 24$)$, pode ser feita por inspeção, conforme descrito em Molina (2011). No entanto, caso existam $i$ e $t$ tais que $\left(c_{2}-\mu_{i t}^{2}\right)<0$, o limitante oferecido por este procedimento de solução será inócuo $(-\infty)$.O valor da variável de transporte para os paletes com custo mais baixo é limitada pelo valor de $R$; assim, para obter um limitante melhor, temos que limitar as variáveis de transporte com custo mais alto. Para melhorar a qualidade dos limitantes inferiores, Norden e Velde (2005) e Molina et al. (2009) propõem limitantes para as variáveis e algumas desigualdades válidas. Devido às extensões propostas no Modelo 1 , no presente artigo algumas adaptações tiveram que ser feitas e são apresentadas resumidamente a seguir:

- Limitante superior para as variáveis $B_{i t}$

- Seja $U D_{i}$ definido como o número de paletes necessários para transportar toda a demanda (i.e., de todos os períodos do horizonte de planejamento) do item $i$, dado pela Equação 26:

$$
U D_{i}=\left\lceil\frac{\sum_{t=1}^{T} d_{i t}}{P_{i}}\right\rceil
$$

- Seja $U C_{i t}$ definido como o número máximo de paletes necessário no período $t$ pelo item $i$, respeitando-se a restrição de capacidade máxima de produção (traduzida em paletes). Este limite é calculado pela expressão (27), obtida a partir das restrições de capacidade (4):

$$
U C_{i t}=\left\lceil\frac{\frac{\mathrm{Cap}_{t}-q_{i}}{b_{i}}}{P_{i}}\right\rceil t=1, \ldots, T ; i=1, \ldots, n
$$

O seguinte limitante superior (28) pode ser estabelecido para as variáveis $B_{i t}$ :

$$
B_{i t} \leq \min \left\{U D_{i}, U C_{i t}\right\} \mathrm{i}=1, \ldots, n ; t=1, \ldots, T
$$

- Desigualdade válida para o problema $\left(z_{1}\right)$

A inequação (29) a seguir garante que, no mínimo, toda a demanda do item $i$ será transportada pelos paletes caros e baratos.

$$
U D_{i} \leq \sum_{t=1}^{T}\left(A_{i t}+B_{i t}\right) \quad i=1, \ldots, n
$$

\section{- Problema de transporte}

Em Norden e Velde (2005) e Molina et al. (2009), o subproblema $\left(z_{2}\right)$ modificado foi resolvido por uma heurística de inspeção. No presente artigo, devido à restrição de não misturar itens diferentes em um mesmo palete, não foi possível adaptar diretamente as heurísticas utilizadas nestes trabalhos anteriores. Ao invés disso, o subproblema $\left(z_{2}\right)$ modificado é interpretado como um problema de transporte, em que as "fontes" ou "centros de produção" (nós de origens) correspondem aos períodos $t=1,2, \ldots, T$, e os "clientes" ou "mercados consumidores" (nós de destinos) correspondem aos itens $i=1,2, \ldots, n$, e com a seguinte interpretação das variáveis:

- $A_{i t}$ - fração da demanda atendida pela fonte $t$ para o cliente $i$ com custo unitário $\left(c_{1}-\mu_{i t}^{2}\right)$;

- $B_{i t}$ - fração da demanda atendida pela fonte $t$ para o cliente $i$ com custo unitário $\left(c_{2}-\mu_{i t}^{2}\right)$.

Para cada fonte $t$, há uma capacidade de oferta, sendo $R$ para as fontes com custos de transporte baixos e min $\left\{U D_{i}, U C_{i t}\right\}$ para as fontes com custos de transporte altos. A Figura 2 ilustra este problema de transporte. Observe na Figura 2 que para cada período $t$ existem duas fontes, a primeira associada ao custo de transporte baixo $\left(c_{1}-\mu_{i t}^{2}\right)$ e a segunda, ao custo de transporte alto $\left(c_{2}-\mu^{2}{ }_{i t}\right)$. Cada fonte pode atender a todos os clientes $i$ (itens). Além disso, dependendo do valor de cada multiplicador Lagrangiano, podem ocorrer casos em que os custos para atender à demanda de alguns itens em alguns períodos sejam negativos. Portanto, este problema de transporte pode ser visto como um caso mais geral do clássico problema de transporte, em que todos os custos entre fontes e clientes são não negativos.

Para resolver esse problema de transporte com possibilidade de custos negativos, a cada iteração, foi usado o pacote CPLEX 10.0. Optou-se pela utilização do CPLEX devido à dificuldade para se resolver problemas de transporte com custos negativos utilizando algoritmos clássicos para problemas de transporte. O desenvolvimento de um

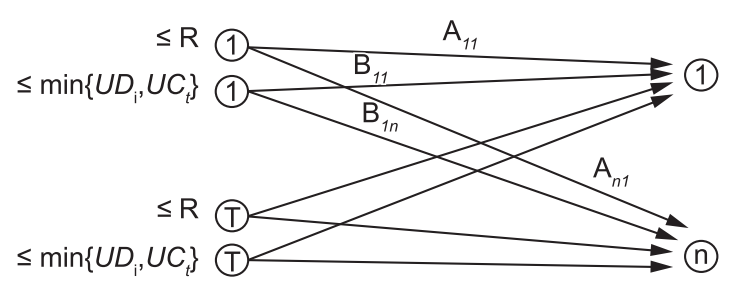

Figura 2. Representação do subproblema de transporte dos itens como um problema de transporte. 
algoritmo específico para o problema em questão é um tópico a ser explorado em pesquisas futuras. Caso a solução encontrada após a resolução dos subproblemas viole as restrições de capacidade (4) em algum período ou as restrições dos paletes (5) para algum item em algum período, é preciso aplicar procedimentos de factibilição. Neste trabalho, utilizaram-se adaptações dos mesmos procedimentos descritos em Molina et al. (2009) - os procedimentos utilizados estão detalhados em Molina (2011).

\subsection{Resultados computacionais: heurística Lagrangiana $\times$ Cplex (1500 segundos)}

A heurística descrita na Seção 4 foi implementada em linguagem $\mathrm{C}$ e foram feitos alguns testes computacionais utilizando os dados gerados de acordo com a descrição feita na Seção 3.1. Nestes testes, foram consideradas apenas as classes de exemplares grandes G1-G16, em que o pacote AMPL/ CPLEX teve mais dificuldades para resolvê-las. Os testes foram realizados no mesmo microcomputador descrito na Seção 3.2 e foi considerado o tempo de 1500 segundos para cada método de resolução (ao invés de 180 segundos, como na Seção 3). Os resultados obtidos foram analisados considerando:

- UBLag: valor da melhor solução factível encontrada pela heurística Lagrangiana;

- LBLag: valor do limitante inferior encontrado pela relaxação Lagrangiana;

- OC: valor da solução obtida pelo pacote CPLEX (com limite de tempo de 1500 segundos);
- TCLag: tempo computacional médio da heurística Lagrangiana;

- GapOLag: valor médio de $100 \times($ UBLag-OC)/ $O C$

- LBgapLag: valor médio de $100 \times($ OC-LBLag)/ $O C$

- GapLag: valor médio de $100 \times($ UBLag-LBLag)/ UBLag.

Na Tabela 9 são apresentados os resultados dos testes realizados. Analisando exclusivamente os resultados da heurística Lagrangiana, a coluna GapLag mostra que, quanto mais apertada a capacidade de produção, maior a dificuldade de resolução da heurística (exemplares das classes G5, G6, G7, G8, G13, G14, G15 e G16). Podemos observar também que, conforme o número de variáveis aumenta, o tempo computacional de resolução do problema utilizado pela heurística também aumenta (coluna TCLag). Isso ocorre pois o problema de transporte (com custos negativos) envolvido em cada iteração da heurística fica mais difícil de ser resolvido. Analisando alguns exemplares da classe G16, foi observado que o pacote CPLEX utiliza em média 15,3 segundos para resolver cada problema de transporte dentro da heurística, o que gera um tempo total de 10.710 segundos. Em outras palavras, aproximadamente $70 \%$ do tempo computacional utilizado para resolver o problema pelo método heurístico é gasto com o pacote de otimização CPLEX. Acreditamos que este tempo possa ser diminuído consideravelmente se o CPLEX for substituído por algum algoritmo específico para resolver o Problema de Transportes com custos negativos.

Tabela 9. Resultados obtidos pelo AMPL/CPLEX 10.0 e a pela heurística Lagrangiana.

\begin{tabular}{|c|c|c|c|c|c|c|}
\hline & \multicolumn{2}{|c|}{ AMPL/CPLEX 10.0 } & \multicolumn{4}{|c|}{ Heurística Lagrangiana } \\
\hline & $T C$ & $\begin{array}{l}\text { Gap } \\
(\%)\end{array}$ & TCLag & $\begin{array}{c}\text { GapOLag } \\
(\%)\end{array}$ & $\begin{array}{c}\text { LBgapLag } \\
(\%)\end{array}$ & $\begin{array}{c}\text { GapLag } \\
(\%)\end{array}$ \\
\hline classe G1 & 1500 & 0,50 & 118,14 & 8,31 & 15,82 & 22,81 \\
\hline classe G2 & 1500 & 0,82 & 849,65 & 8,19 & 15,73 & 22,63 \\
\hline classe G3 & 1500 & 0,65 & 1178,28 & 8,84 & 14,72 & 22,26 \\
\hline classe G4 & 1500 & 1,01 & 1500,00 & 8,17 & 16,07 & 22,93 \\
\hline classe G5 & 1500 & 1,62 & 269,71 & 39,32 & 37,45 & 62,04 \\
\hline classe G6 & 1500 & 2,28 & 1500,00 & 37,31 & 29,23 & 55,63 \\
\hline classe G7 & 1500 & 2,19 & 1500,00 & 38,86 & 33,24 & 59,18 \\
\hline classe G8 & 1500 & 3,57 & 1500,00 & 23,18 & 32,09 & 47,83 \\
\hline classe G9 & 1500 & 1,06 & 124,72 & 11,99 & 10,14 & 20,92 \\
\hline classe G10 & 1500 & 1,64 & 932,17 & 11,35 & 10,23 & 20,42 \\
\hline classe G11 & 1500 & 1,21 & 1240,38 & 11,77 & 9,63 & 20,27 \\
\hline classe G12 & 1500 & 6,58 & 1500,00 & 7,06 & 14,75 & 20,77 \\
\hline classe G13 & 1500 & 2,01 & 272,84 & 29,40 & 23,55 & 46,03 \\
\hline classe G14 & 1500 & 2,37 & 1500,00 & 25,99 & 18,21 & 39,47 \\
\hline classe G15 & 1500 & 2,36 & 1500,00 & 30,12 & 19,57 & 43,80 \\
\hline classe G16 & 1500 & 4,93 & 1500,00 & 18,79 & 20,45 & 35,40 \\
\hline
\end{tabular}


Analisando os resultados do CPLEX para resolver o Modelo 1, tem-se que o pacote de otimização utiliza o tempo máximo disponível (1500 segundos) e não consegue provar a otimalidade. No entanto, o Gap é reduzido pela metade quando comparamos com os resultados referentes ao limite de tempo de 180 segundos e, além disso, com o tempo adicional, o pacote de otimização obteve uma solução factível para todas as classes testadas. Ao comparar os resultados obtidos pelo pacote com aqueles obtidos pela heurística, tem-se que a solução factível encontrada pelo pacote em geral é melhor do que a solução factível da heurística. Conclui-se que a heurística (em sua versão atual) não é competitiva com o CPLEX nestas classes de problemas.

\section{Considerações finais e trabalhos futuros}

Neste trabalho, foram apresentados três modelos matemáticos para o problema de dimensionamento de lotes com custos de transporte, considerando diferentes situações que ocorrem na prática. Os modelos são uma alternativa para resolver problemas realistas de dimensões moderadas, pois em todos os casos o AMPL/CPLEX resolve os modelos em um tempo computacional razoavelmente pequeno (180 segundos) e com valor muito próximo da otimalidade. Quando os problemas possuem dimensões maiores, o pacote AMPL/CPLEX tem dificuldades para obter soluções factíveis com pequenos GAPs de otimalidade dentro deste tempo limite, principalmente para resolver o Modelo 1. Porém, aumentando-se o limite de tempo para valores maiores, mas ainda aceitáveis na prática para decisões de médio prazo (1500 segundos), o CPLEX foi capaz de obter soluções factíveis com GAPs relativamente pequenos.

Os Modelos 2 e 3 foram mais fáceis de serem resolvidos pelo CPLEX para as classes de exemplares analisadas, pois além de terem os menores GAPs, alguns exemplares foram resolvidos provando a otimalidade da solução. Isto pode ter ocorrido devido às novas restrições dos modelos que podem gerar planos de corte mais eficientes para a resolução dos problemas. Como uma alternativa ao pacote comercial para resolver o Modelo 1, foi desenvolvida uma heurística Lagrangiana, com a qual foram encontradas soluções viáveis para todos os exemplares testados. Porém, nestes testes realizados, o desempenho da heurística (na sua versão atual) foi dominado pelo CPLEX.

Alguns estudos estão na nossa agenda de pesquisa. Por exemplo, pretendemos realizar experimentos computacionais adicionais com os Modelos 2 e 3, para verificar seus comportamentos e limitações para outras classes de problemas, em particular para diferentes valores do número máximo de caminhões mais baratos que podem ser utilizados. Também pretendemos estudar um método específico de resolução do caso mais geral do problema de transporte em que são admitidos custos negativos, para reduzir os tempos computacionais requeridos pela heurística Lagrangiana.

Outra perspectiva interessante para pesquisa futura seria estudar a variação do problema integrado em que os itens são colocados nos caminhões sem a utilização de paletes, o que também é comum na prática. Neste caso, o empacotamento não poderá ser simplesmente tratado num pré-processamento, resolvendo-se a priori problemas de carregamento de paletes do produtor, pois depende da decisão do dimensionamento de lotes a respeito de quais itens serão empacotados. Isto deve dificultar significativamente a resolução do problema. Outro trabalho futuro interessante seria estudar a extensão do problema integrado em que são considerados vários armazéns ou clientes dispersos geograficamente para a distribuição da produção. Neste caso, junto ao problema de dimensionamento de lotes, deve ser combinado um problema de roteirização dos veículos para a distribuição.

\section{Agradecimentos}

Os autores agradecem aos dois revisores anônimos pelos úteis comentários e sugestões. Esta pesquisa contou com apoio financeiro da FAPESP, CAPES e CNPq.

\section{Referências}

BERTAZZI, L.; SPERANZA, M. G. Models and algorithms for the minimization of inventory and transportation costs: a survey. In: SPERANZA, M. G.; STAEHLY, P. (Ed.). New trends in distribution logistics. Lecture notes in economics and mathematical systems. Berlin Heidelberg: Springer-Verlag, 1999. v. 480, p. 137-157.

BIRGIN, E. G.; LOBATO, R. D.; MORABITO, R. An effective recursive partitioning approach for the packing of identical rectangles in a rectangle. Journal of the Operational Research Society, v. 61, 306-320, 2010.

BRAHIMI, N. et al. Single item lot sizing problems. European Journal of Operational Research, v. 168, p. 1-16, 2006.

DREXL, A.; KIMMS, A. Lot sizing and scheduling - survey and extentions. European Journal of Operational Research, v. 99, p. 221-235, 1997.

ERENGUÇ, S. S.; SIMPSON, N. C.; VAKHARIA, A. J. Integrated production/distribution planning in supply chains? An invited review. European Journal of Operational Research, v. 115, p. 219-236, 1999.

JANS, R.; DEGRAEVE, Z. Meta-heuristics for dynamic lot sizing: a review and comparison of solution approaches. European Journal of Operational Research, v. 177, p. 1855-1875, 2007.

JANS, R.; DEGRAEVE, Z. Modelling industrial lot sizing problems: a review. International Journal of Production Research, v. 46, n. 6, p. 1619-1643, 2008. 
KARIMI, B.; GHOMI, S. M. T. F.; WILSON, J. M. The capacitated lot sizing problem: a review of models and algorithms. OMEGA, v. 31, p. 365-378, 2003.

LEE, W.-S.; HAN, J. H.; CHO, S. J. A heuristic for a multi-product dynamic lot-sizing and shipping problem. International Journal of Production Economics, v. 98, p. 204-214, 2005.

MOLINA, F. Heurísticas para um problema de dimensionamento de lotes com restrições de capacidade e custos de transporte. Dissertação (Mestrado)-Universidade Estadual Paulista, São José do Rio Preto, 2007.

MOLINA, F. Modelos e métodos para problemas de dimensionamento de lotes com restrições e decisões de distribuição. Tese (Doutorado em Engenharia de Produção)-Universidade Federal de São Carlos, São Carlos, 2011.

MOLINA, F. et al. An approach using Lagrangean/surrogate relaxation for lot-sizing with transportation costs. Pesquisa Operacional, v. 28, n. 2, p. 269-288, 2009.

MORABITO, R.; PUREZA, V. Loading optimization. In: ERDOGDU, F. (Org.). Optimization in Food Engineering. Boca Raton: CRC Press; Taylor \& Francis Group, 2008. p. 657-683.

NORDEN, L.; VELDE, S. Multi-Product lot-sizing a transportation capacity reservation contract. European Journal of Operational Research, v. 165, p. 127-138, 2005.

OLIVEIRA, L. K.; MORABITO, R. Métodos exatos baseados em relaxações Lagrangiana e surrogate para o problema de carregamento de paletes do produtor. Pesquisa Operacional, v. 26, n. 2, p. 403-432, 2006.

RIZK, N.; MARTEL, A. Supply chain flow planning methods: a review of the lot-sizing literature. Working Paper DT-2001-AM-1, Université Laval, QC, Canada, 2001.

ROBINSON, P.; NARAYANANB, A.; SAHINC, F. Coordinated deterministic dynamic demand lot-sizing problem: a review of models and algorithms. Omega, v. 37, p. 3-15, 2009.

SENNE, E. L. F.; LORENA, L. A. N. Lagrangean/surrogate heuristics for p-median problems. In: LAGUNA, M.; GONZALEZ-VELARDE, J. L. (Ed.). Computing tools for modeling optimization and simulation: interfaces in computer science and operations research. Kluwer Academic Publishers, 2000. p. 115-130.

TOLEDO, F. M. B.; ARMENTANO, V.A. A Lagrangianbased heuristic for the capacitated lot-sizing problem in parallel machines. European Journal of Operational Research, v. 175, p. 1070-1083, 2006.

TRIGEIRO, W. W.; THOMAS, L. J.; McCLAIN, J. O. Capacitated lot sizing with setup times. Management Science, v. 35, n. 3, p. 353-366, 1989.

VROBLEFSKI, M.; RAMESH, R.; ZIONTS, S. Efficient lot-sizing under a differential transportation cost scr serially distributes ware-houses. European Journal of Operational Research, v. 127, p. 574-593, 2000.

ZANGWILL, W. L. A backlogging model and a multiechelon model of a dynamic economic lot size production system - a network approach. Management Science, v. 15 , n. 9 , p. $506-527,1969$. 